\title{
EXPERIMENTAL STUDIES ON FATIGUE RESISTANT PERFORMANCE OF EXPANSION JOINT SYSTEM
}

\author{
By Matsuji SASADO*, Kyuichi MARUYAMA**, Kenji HAYASHI*** \\ and Hirokazu FUJII****
}

\begin{abstract}
It is a general trend to make maintenance free designs for bridge structures. Although the design concept of expansion joints was different from that of bridge structures so far, it will be required to take an expansion joint as a structural element of a bridge as long as the maintenance-free concept is applied to bridge designs.

It is discussed in this paper how expansion joints should be designed and what problems will arise under the maintenance free concept. An experimental study was conducted on a specific expansion joint system (mechanical expansion joint system). Through fatigue tests, several material and structural problems in a joint system were pointed out. Finally, those defects were removed and an improved joint system was proposed.
\end{abstract}

\section{INTRODUCTION}

In these years, bridge structures have become larger in size and more complicated in structural design. They are also required to serve under severer environmental conditions than used to be. Accordingly, it has become more necessary to understand bridges' structural behaviors in detail under various conditions. Since an expansion joint system is also one of the structural elements of a bridge, it is important to clarify its structural characteristics under various conditions.

Expansion joint system may generally be classifified into a metal type, a rubber type and a composite type which is the combination of the first two types. For selecting an expansion joint system, it should be basically considered how a highway is charaterized, what features a bridge have, and how much the amount of expansion is required ${ }^{1,2)}$.

In highway bridge design practice, the effects of fatigue due to repeated loadings are not generally considered. As for steel plate floors of bridges, however, such effects must be examined on a collateral basis. Because these floors are liable to be affected by fatigue since stresses due to their dead loads account for rather a small portion of their design stresses and higher stresses due to live loads having large amplitude are produced repetitively ${ }^{1)}$.

Expansion joint systems, which are directly subjected to repeated traffic loads are the parts most suscreptible to damage among all bridge structural elements. Moreover, stresses caused by live loads account for a larger portion of design stresses in expansion joint system than in steel plate floors. Thus, it is important to clarify the fatigue characteristics of expansion joint system.

\footnotetext{
* Member of JSCE, Dr. Eng., Prof. of the Technological Univ. of Nagaoka (Kamitomioka Nagaoka)

** Member of JSCE, Ph. D., Assoc. Prof. of the Technological Univ. of Nagaoka (ditto)

*** Member of JSCE, M. Eng., Research Assoc. of the Technological Univ. of Nagaoka (ditto)

**** Member of JSCE, Nitta Industries Corporation (Yamatokooriyama Nara)
} 
The basic attitude in this study is to introduce a maintenance-free concept into expansion joint systems and to develop criteria for such systems. The expansion joint systems to be studied has mechanical expansion devices. The experiment has done to find how the system behaves under static and dynamic loadings. In process of test, several defects were revealed in view of maintenance-free. It is another objective of this study to solve such problems and to improve the expansion joint systems.

\section{GENERAL SCOPE OF THE PROPOSED EXPANSION JOINT SYSTEM}

Expansion and contraction in this expansion joint system depend on mechanical device which allows the interspace between the two middle beems to widen up or narrow down keeping the middle beem in parallel to each other. In addition, rubber seals are fastened by mechanical means to insure perfect surface drainage. The system can be designed to deal with a greater amount of expansion movemen' by increasing the number of cells (i.e., rubber seals). Described in the following paragraphs are the load support/transfer mechanism, the expansion/contraction mechanism and the waterproof device of this joint system.

\section{(1) Load support/transfer mechanism}

The wheel load of moving vehicles is firstly transferred to the middle beams (hot-rolled atmospheric corrosion resisting steel for welded structures, SMA 50) which are placed parallel to the interspace between the ends of bridge girders. Then, the load on the middle beams is carried to the support beams (built-up $\mathrm{H}$-shaped members, SM 50) which are spaned in direction of the bridge axis. Finally, the load is transfered from the support beams to the floor decks and slabs by way of the slide bearings (See Fig. 1). Slide bearings and shock absorbers are made of elastomer and designed to absorb and alleviate impact loads.
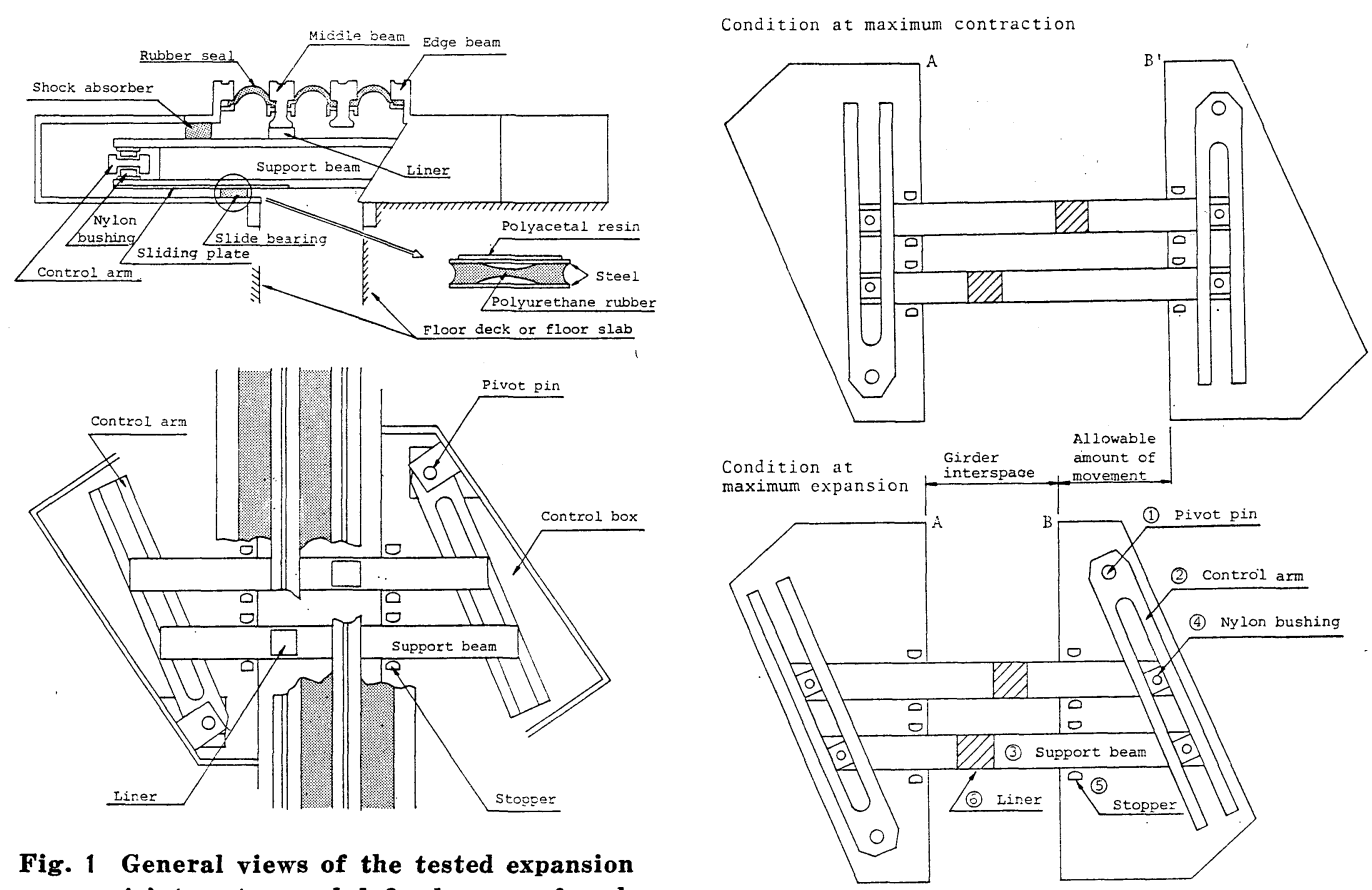

Fig. 1 General views of the tested expansion joint system and defined name of each component

Fig. 2 Expansion/contraction mechanism 


\section{(2) Expansion/contraction mechanism}

Expansion and contraction in this system take place in the following sequence (See Fig. 2).

a) By contraction (or expansion) of the bridge girders, the girders interspace $A B$ shifts to $A B$ '.

b) The control arm (2) rotates about the pivot pin (1).

c) The nylon bushing (4) attached to the tip of the support beam (3) slides along the groove in the control arm.

d) The support beam moves in the direction of a bridge axis.

e) At this time, the support beam can move only in the direction of a bridge axis by the stopper (5).

f) Thus, the liner (6) fixed to the support beam is also movable in the direction of a bridge axis only.

g) Naturally, the middle beams fixed to the liner makes parallel movement to each other in the direction of the bridge axis.

The slide bearings are composed by polyacetal resin, steel and polyurethane rubber as shown in Fig. 1. Tortional movement of the girder interface is absorbed by the slide bearings and shock absorbers, both of which are made of elastomer (polyurethane).

\section{(3) Waterproof device}

A monolithic rubber seal (made of polychloroprene rubber) is fastened to an edge beam by a L-bar and machine bolts, and is fastened to a middle beam by a T-bar and machine bolts, as shown in Fig. 3. Accordingly, setting or removing these rubber seals can be made by tightening or loosening the bolts as appropriate.

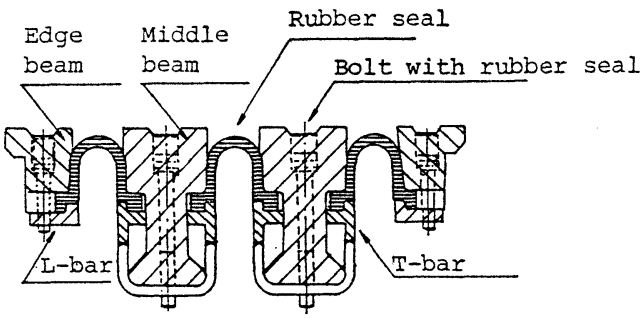

Fig. 3 Waterproof device

\section{EXPERIMENTAL STUDY}

\section{(1) Purpose}

No experimental study have been conducted concerning this type of expansion joints. Accordingly, the experiments on strength performance of this expansion joint system were carried out to make examinations of the following; firstly, to find out how the load imposed on the system would be transferred to each part of the system, and secondly, to investigate the behavior of each part when the system was subjected to the repeated loads. Requirements for durability of the system were to be made step by step in the process of experimental tests.

The static loading test is first scheduled to examine the calculated results for designing the joint system. Next, in the fatigue tests, dynamic behaviors of the elements of the joint system and the interaction of elements are studied. As the joint system is to be designed on maintenance-free concept, the fatigue performance should be investigated as to the elements of the system, such as the middle 
beams, the support beams, the welds in the liners which connect the foregoing two types of beams, the slide bearings and the shock absorbers.

The basic intent of this study is to develop the expansion joint system with sufficient fatigue resistant performance. After various improvements being done, the revised joint system is proposed.

\section{(2) Test procedure}

\section{a) Materials and test specimens}

The middle and edge beams are made of SMA 50 steel; support beams, of SM 50 ; sliding plates, of SUS 304 stainless steel; and other members of SS 41 steel. Further, polyurethane rubber (U) and polyacetal resin (POM) are used for the slide bearings and polychloroprene rubber (CR), for rubber seals. The test specimen consists of two middle beams and two edge beams. The length of each beam is $300 \mathrm{~cm}$. The sectional properties of the middle and support beams are shown in Fig. 4 (a) and (b).

\section{b) Applied loading}

It is a general practice to assure that a rear wheel load of $\mathrm{T}-20$ acts on the system as a concentrated load, with the impact coefficient taken as $100 \%{ }^{2}$. Accordingly, the design load for this expansion joint system is also taken as a wheel load of 8 tons, with impact coefficient of $100 \%$ being considered. Providing that the wheel contacts on the road with $200 \mathrm{~mm}$ length, a middle beam in the system with three more cells takes full wheel load on it when the interspace between middle beams gets wide at maximum (shown in Fig. 5).

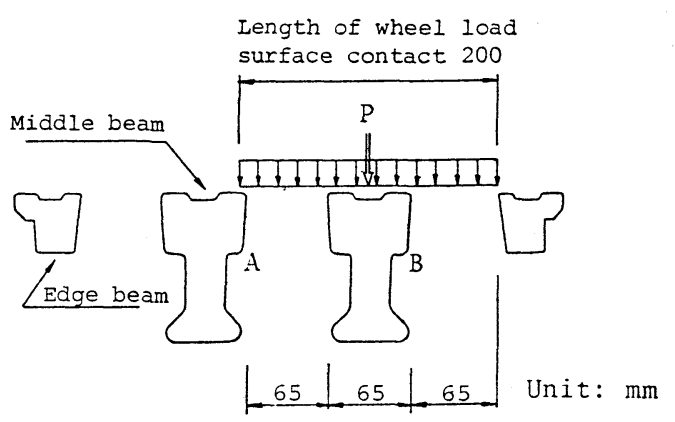

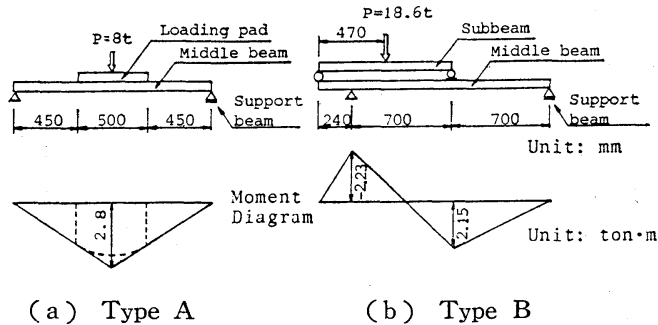

Fig. 6 Pattern of applied loadings

\section{Fig. 5 Wheel load (P) acting on a middle beam (B)}

The wheel load acting on the middle beam may be taken as 13 tons including the impact load. In order to determine the loading patten and the amplitude of applied load, the maximum positive and negative bending moments calculated in the actual expansion joint system should be taken as the basic index. The test load has been established in such a way that the maximum bending moment produced in a test specimen should be equivalent to that in a whole system of the actual expansion joints. The type (A) loading pattern as shown in Fig. 6 (a) has been adopted for providing the test data which should be particularly helpful in ascertaining the behavior of the system as a whole. On the other hand, the type (B) loading pattern as shown in Fig. 6 (b) has been employed to obtain the test data which may serve the purpose of improving the design of the built-up support beams and the fatigue resistant performance of the slide bearings. Furthermore, the stress acting on the section of support beams can be estimated in safety by applying above loads to the test specimen.

Concerning with fatigue strength of the expansion joint system, it should be noticed that the current specifications for highway bridges do not tell either what type of load should be considered for fatigue test, or what criteria should be applied as to the allowable stresses of materials. In the case of the steel plate floors, it is specified that one truck load (T-load) without an impact coefficient is used for the examination purpose and that the stress amplitude which dose not result in fatigue-caused cracks under 2-million cycles of load repetition is taken as a standard. 
As the fatigue test load, the whole load with an impact coefficient as $100 \%$ was applied under following considerations.

(1) the portion of the live load to the total design load is larger in the expansion joint system than steel plate floor.

(2) the expansion joint are apt to take impact loads directly by moving vehicles.

As the design criteria, the followings were adopted.

(1) steel elements of the expansion joint system should withstand 2-million applications of the repeated load as required in the steel plate floor design.

(2) the rubber elements should meet the applicable peeling-resistive requirements.

c) Equipment and mesurement

The tests were conducted by imposing static or dynamic loads on the specimen by utilizing the reaction floor, the reaction frame and the actuator (developing dynamic loads +30 tons to -30 tons). The fatigue tests were conduced by pulsating the specimens, in one direction, with the oscillating frequency maintained at $2 \sim 3 \mathrm{~Hz}$. For type A loading patten, the size of loading plate was determined to the same width of contact area of a wheel on the roadsurface, such as $500 \mathrm{~mm}$. The loading plate was made of polychloroprene rubber. For type B loading pattern, a subbeam with a span $940 \mathrm{~mm}$ was set to transfer the actuator face to a test specimen by two concentrated loadings. The scene of the fatigue test by Type B loading is shown in Fig. 7.

In order to check the calculated results by elastic analysis, strain gauges were attached to the upper and lower flange surfaces at the midspan, and the quarter-span points. Recording and processing were made by an automatic measurement system which consists of a multipoint digital recorder, a micro-computer, a $\mathrm{X}-\mathrm{Y}$ plotter and so on.

In the fatigue tests, the fatigue-caused cracks

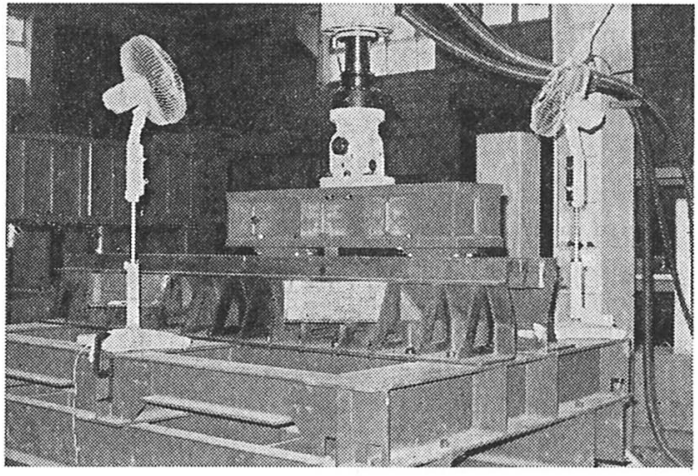

Fig. 7 Fatigue test under type B loading developed in the middle and support beams and in the welds of the liner were detected by visual inspection and by color check. Peeling of the slide bearing (polyurethane rubber) was detected by visual inspection alone.

\section{TEST RESULTS}

\section{(1) Behaviors under static loading}

Where the load applied by type A pattern, the measured values of strain were compared with the corresponding computed values which were obtained by the use of a simple grid theory in which it was assumed that only shear force was transferred at the joint between the middle beam and the support beam. In computation the load was treated in two ways. One was concentrated load. The other was partially distributed load which looked to represent the actual condition. The comparison of these two cases indicates that the assumption of concentrated loading in calculation represents the test results reasonably. Therefore, further comparisons and discussions are made on the base of concentrated loading in analysis

In the cases of both type $A$ and type $B$ loading, the computed values of strain coincide reasonably with the measured values. Therefore, the computation by a simple grid theory could be effective for evaluation of the fatigue performance of the joint system. 


\section{(2) Fatigue test under type A loading}

The load ( $P=8$ tons) as shown in Fig. 6 (a) was applied repeatedly to the specimen in which bolt holes for installation of rubber seals had been drilled at $30 \mathrm{~cm}$ intervals. In consequence, a crack developed in the bolt hole on the lower flange surface near the midspan of the middle beam when the load had been repeated 380-thousand times, and the failure of the beam followed.

The bending moment at the midspan of the girder (i.e., the middle beam) can be calculated as $2.8 \mathrm{ton} \cdot \mathrm{m}$ and this would caused the stress of $24.7 \mathrm{~kg} / \mathrm{mm}^{2}$ (which was larger than the allowable stress $\sigma_{a}=21 \mathrm{~kg} / \mathrm{mm}^{2}$ ) in the edge of the lower flange of the beam. Also, judging from the measurement data, the stress which actually developed in the girder should be $23.7 \mathrm{~kg} / \mathrm{mm}^{2}$. This means that the girder was subjected to the stress which exceeded the allowable value. Further, when the loss of sectional area by bolt holes and the stress concentration region are taken into account, it can be considered that the stress around the bolt holes in the girder exceeded the yield stress $\left(\sigma_{y}=38 \mathrm{~kg} / \mathrm{mm}^{2}\right)$ of steel.

It is presumed that few cases can be found that the fatigue strength of thick steel plates like the middle beams was ascertained by the experimental means. However, reference to Fatigue Data No. 67 (Reference No. 3) makes it possible to interpret that the middle beams without bolt hole can withstand more than 800-thousand applications of the repeated load ( $P=12$ tons) which produces yield stress at the extreme fiber in the lower flange at the midspan of the middle beam (See Fig. 8).

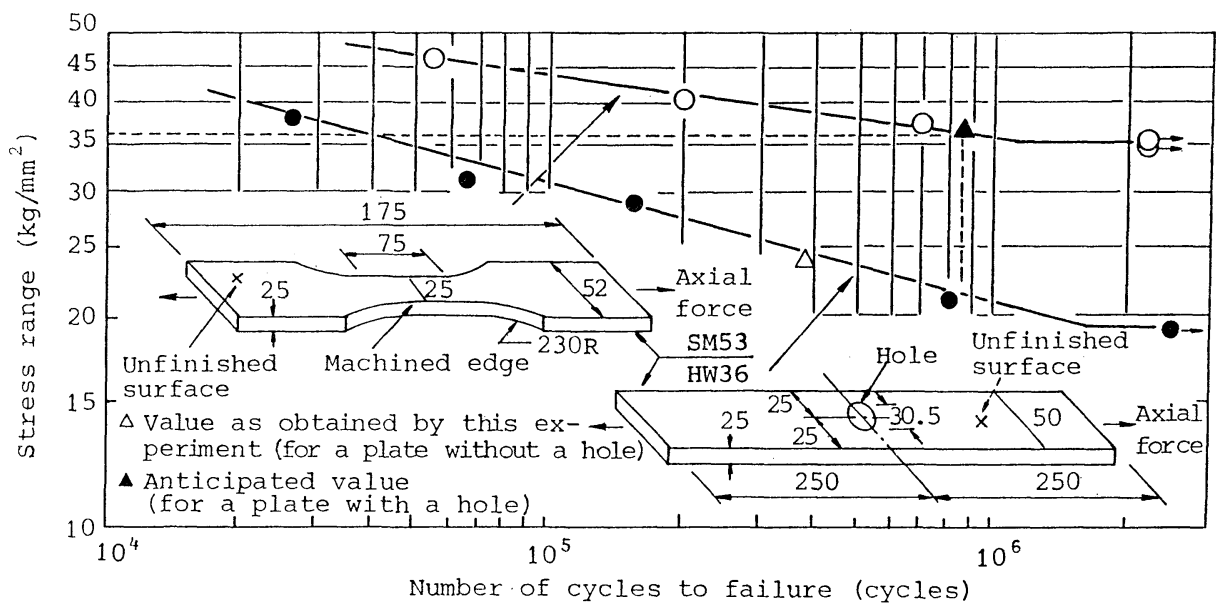

Fig. 8 S-N curves for plates with and without hole

Hence, another fatigue test was conducted to make sure the above interpretation. A load of 12 tons instead of 8 tons (See Fig. 6 (a)), was applied to the beam without holes for installation of rubber seals. In this case, no fatigue crack was initiated in the beam itself at 1.6-million cycles. However, a crack began to develop in the weld of the liner at the overhang side of the middle beam, and this was soon followed by the failure of the beam (See Fig. 9).

In consideration of these test results, the bolt

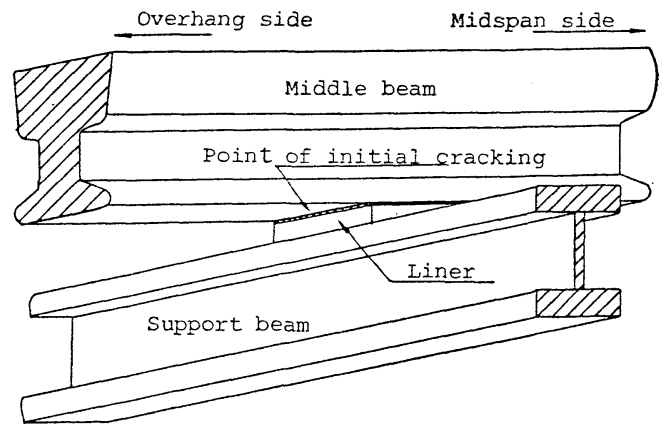

Fig. 9 Crack initiation at welded part in liner holes of the middle beam for installation of rubber seals were relocated from the previous $300 \mathrm{~mm}$ spacing to the new positions which are at a distance of $250 \mathrm{~mm}$ from the support beam toward the 
midspan of the middle beam (Consequently, the bolt hole spacing at the midspan portion of the middle beam became $900 \mathrm{~mm}$ ). Also, a specimen with new bolt hole positions was subjected to the dynamic waterproof test, and it was ascertained that the waterproof performance of the joint was not deteriorated by the change in the bolt hole positions. The detail of such hydraulic test, however, will be omitted in this paper.

The position at which each middle beam is to be welded for interconnection was so selected as to keep the bending moments at that position relatively small under various loading conditions; thus, the position $175 \mathrm{~mm}$ toward the midspan of the middle beam from the point right above the support beam (i.e., a point at a $1 / 8$ of the span $l$ or $1 / 8 l$ point) was determined. For the interconnection, the full penetration butt weld was used.

\section{(3) Fatigue test under type $B$ loading}

Whereas the discussion in the Type A loading test described in the preceding paragraph was forcused on the investigation of the fatigue characteristics of the middle beam itself, the objectives of the Type B loading test were primarily intended for the investigation of the fatigue characteristics of the actual joint system as a whole. Thus, in the test, not only the maximum positive and negative bending moments acting on the middle beam was applied to the girder but also the pattern of moment distribution was made to correspond with the bending moment diagram of the actual expansion joint system. Also an attempt was made to check and verify through the test if the positions of bolt holes in the middle beam for rubber seals as determined in the preceding paragraph was really reasonable.

The repeated load was imposed on the specimen as shown in Fig. 6 (b). As the result, at 1.28million cycles fatigue cracks began to develop in the weld which joined the web with the upper flange of the support beam as shown in Fig. 10, and in the part which a liner was welded to a middle beam, and further peeling of polyurethane rubber of the slide bearing was observed at this stage. No crack development, however, was observed in the weld interconnecting the elements of middle beams and near the bolt holes of the middle beam.

In order to solve the above problems, the improvements of the materials and construction were discussed in the following subparagraph.

\section{a) Design of built-up support beams}

It was presumed that the crack initiation from the toe of the part which a fillet weld was returned continuously around the corner of the web of support beam was attributable to the fact that the support beam could not maintain enough strength to carry indirectly the reaction of the slide bearing by way of two nylon bushings and a control arm when the interspace between the middle beams became the greatest. Because at such time the slide bearing was at a position without the web of the support beam as can be seen in Fig. 10. Namely, the upper and lower flanges overhanging from the web behaved like cantilevered plates. With repetition of loading the corner of the upper flange-to-web connection was in particular subjected to repeated tensile stress caused by the bending moment. To overcome this problem, the web was extended on the slide bearing (in Fig. 10), and the fatigue test by the Type $B$ loading was conducted

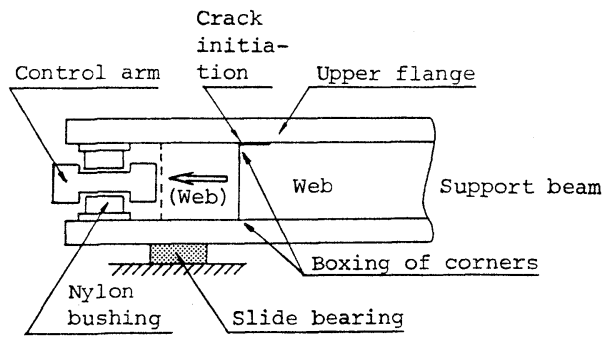

Fig. 10 Crack initiation in a support beam on the redesigned specimen. This time, no fatigue was seen to develop on the support beam at more than 2-million cycles. It was verified that the improvement adopted as above was proper and reasonable.

It is presumed that the fatigue resistant performance of the support beam will be further improved if the web edges are rounded; however, since the redesign as described above is believed to suffice the 
purpose (the boxing zone being subjected to very low stresses), the rounding of web is not an essential requisite, particularly when the economy and construction feasibility are taken into account.

\section{b) Liner welding method}

Full round fillet welding was used to install the liner by which the middle beam are joined across to the support beam. It should be noted here that careful attention must be paid to the welding of the liners since the stress concentration due to structural discontinuity took place at the welds in the liners when the shear forces in the middle beams were transferred to the support beam through these liners. In addition, the welds tended to become brittle by the adverse heat effects caused by welding performed both from the middle beam side and from the support beam side. There also existed the residual stresses in these specific welds. Further, it needs to be mentioned that highly skilled welding technique was required wherever a welding lines traversed the corners of the liners because the welding position had to be changed from the horizontal to the overhead at such locations.

Thus, in order to enable the boxing in the upper and lower sides of four corners of the liner which was attached to the middle beam and the support beam to be performed in one continuous operation, $7 \mathrm{~mm}$ bevels were provided at all member edges before and behind, to the right and left of, and above and below the liners. The fatigue tests conducted by the use of the two specimens made in the manner as described above led to the results that the first and the second specimens were ruptured by fatigue in the weld at 1.5-million cycles and 3.62-million cycles respectively. This rather pronounced variation in the test results indicates that the fatigue strength of this type of members is greatly affected by the quality of welds, and this leads to an assertion that sufficient quality control of the work is considered essential. It is presumed that the fatigue strength can be further improved if finish by grinder of the terminal ends of welds is included in the improvement method described above.

\section{c) Examination of slide bearings}

Although the real cause of the rupture (peeling or cracking at bonded points) of polyurethane rubber used in slide bearings could not be clarified precisely, it was decided to be studied, in consideration of stressconcentration and deformation capacity, six type of slide bearings, i.e., types (a) through (f) asshown in Fig. 11. The test resulted in the finding that only Type (a) was capable of withstanding more than 2-million applications of the repeated load without development of peeling or cracks as indicated in Table 1. A comparison of Type (a) with Type (b) indicated that the fatigue resistant performance of the slide bearing was greatly affected by the hardness of rubber. Comparing Type (b) with Type (d), it was recognized that such performance was greatly influenced also by the shape of the bearing.

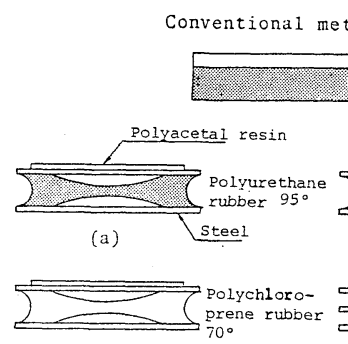

(c)

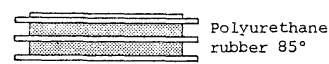

(e)

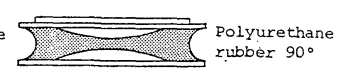

(b)

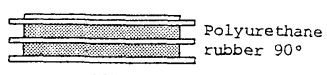

(d)

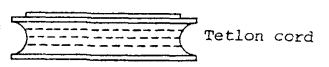

(f)
Table 1 Fatigue resistant performance of slide bearings

\begin{tabular}{|c|c|c|}
\hline Types & $\begin{array}{c}\text { Number of cycles } \\
\text { to failure } \\
(\times 10 \text { thousand cycles })\end{array}$ & Remarks \\
\hline $\mathrm{a}$ & 250.0 & No peeling or crack observed \\
\hline $\mathrm{b}$ & 5.0 & $\begin{array}{c}\text { Crack initiation recognized } \\
\text { in polyurethane rubber }\end{array}$ \\
\hline $\mathrm{c}$ & 72.5 & $\begin{array}{c}\text { Crack initiation recognized } \\
\text { in polychloroprene rubber }\end{array}$ \\
\hline $\mathrm{d}$ & 2.4 & $\begin{array}{c}\text { Peeling recognized in } \\
\text { the bonded interface }\end{array}$ \\
\hline $\mathrm{e}$ & 17.5 & $\begin{array}{c}\text { Peeling recognized in } \\
\text { the bonded interface }\end{array}$ \\
\hline $\mathrm{f}$ & 51.2 & Crack recognized in 1anination \\
\hline
\end{tabular}

Fig. 11 Various types of slide bearing

\section{d) Fatigue resistant performance of improved expansion joint system}

In order to prove the fatigue resistant performance of the improved expansion joint system, three test specimens were manufactured and subjected to the fatigue tests using the type B loading method. 
From the tests, it was found that all of them were capable of withstanding more than 2-million applications of the repeated load without developing cracks or peeling in any parts except in the welds of the liners (See $(\mathbf{3})-\mathbf{b})$ ). Thus, it is believed that the reasonableness of the adopted improvement method was verified well by the test results. As for the methods of welding the liners, it should be necessary to make further studies in detail. Especially, the need of sufficient quality control of the work is keenly felt.

\section{CONCLUDING REMARKS}

The experimental studies herein described heve been intended to find out the potential problems in expansion joint systems when the maintenance-free design concept is introduced into them. Using the specimens of a specific type of the joint system, discussion were excuted to countermeasures to solve such problems, and evaluation for them experimentally through the fatigue tests. Also, the studies have been intended to make a guide line which indicates how the test loads should be treated and what should be used as criteria for judgement when the fatigue of expansion joint systems has to be examined on a collateral basis.

Despite the fact that expansion joint systems are more liable to be damaged by fatigue than any other sort of bridge structural elements, almost no documented record of research studies on the fatigue resistant performance of the joint system was available as far as the authors were able to know by their fairly extensive literature retrieval efforts. When the maintenance-free concept is applied to the bridge structure as a whole, and, when the more rational design of expansion joint systems is requested, the fatigue resistant performance of expansion joint systems becomes a crucial point. The authors will be pleased if the experimental studies reported in this paper is useful as an example of the research on this specific subject.

In addition, in order to investigate the conditions of concrete deposited to the bottom of the control box, an experimental construction trial was conducted by injecting grout a simulated control box made by acrylic resin for this specific purpose. It was verified by the result of this experiment that the condition of deposit was highly satisfactory.

\section{ACKNOWLEDGMENTS}

The authors wish to express their great indebtedness to Dr. Atsushi HIRAI, Prof. Emeritus, Tokyo University and Technological University of Nagaoka for his guiding advice; to Mr. Yugo NAKAMURA, technical official, and other staff members of Structure Seminar of Technological University of Nagaoka and also to the staff members of Nitta Industries Corporation, Construction Materials Department for their help and assistance given to the authors in he course of the experiments.

\section{References}

1) Specifications for Highway Bridge and Commentary on the Spec. by Japan Road Association, 1980.

2) Manual on Bridge Expansion Joint Systems by Japan Road Association, 1970.

3) Fatigue Data Sheets by Society of Steel Construction of Japan. 